\title{
SDN Based User-Centric Framework for Heterogeneous Wireless Networks
}

\author{
Zhaoming Lu, ${ }^{1,2}$ Tao Lei, ${ }^{1,2}$ Xiangming Wen, ${ }^{1,2}$ Luhan Wang, ${ }^{1,2}$ and Xin Chen ${ }^{1,2}$ \\ ${ }^{1}$ Beijing Key Laboratory of Network System Architecture and Convergence, Beijing University of Posts and Telecommunications, \\ Beijing 100876, China \\ ${ }^{2}$ Beijing Laboratory of Advanced Information Networks, Beijing University of Posts and Telecommunications, Beijing 100876, China \\ Correspondence should be addressed to Zhaoming Lu; lzy_0372@163.com
}

Received 5 February 2016; Accepted 23 June 2016

Academic Editor: Young-June Choi

Copyright (C) 2016 Zhaoming Lu et al. This is an open access article distributed under the Creative Commons Attribution License, which permits unrestricted use, distribution, and reproduction in any medium, provided the original work is properly cited.

\begin{abstract}
Due to the rapid growth of mobile data traffic, more and more basestations and access points (APs) have been densely deployed to provide users with ubiquitous network access, which make current wireless network a complex heterogeneous network (HetNet). However, traditional wireless networks are designed with network-centric approaches where different networks have different quality of service (QoS) strategies and cannot easily cooperate with each other to serve network users. Massive network infrastructures could not assure users perceived network and service quality, which is an indisputable fact. To address this issue, we design a new framework for heterogeneous wireless networks with the principle of user-centricity, refactoring the network from users' perspective to suffice their requirements and preferences. Different from network-centric approaches, the proposed framework takes advantage of Software Defined Networking (SDN) and virtualization technology, which will bring better perceived services quality for wireless network users. In the proposed user-centric framework, control plane and data plane are decoupled to manage the HetNets in a flexible and coadjutant way, and resource virtualization technology is introduced to abstract physical resources of HetNets into unified virtualized resources. Hence, ubiquitous and undifferentiated network connectivity and QoE (quality of experience) driven fine-grained resource management could be achieved for wireless network users.
\end{abstract}

\section{Introduction}

Wireless networks are undergoing a bold change. Increasing data traffic pours into wireless networks from wired networks, in which video streaming traffic is the main force. According to the report of Ericsson [1], mobile data traffic is expected to grow at a CAGR (Compound Average Growth Rate) of around 45 percent (2013-2019). This will result in a traffic increase of around 10 times by the end of 2019 . In order to accommodate the explosive mobile data traffic growth and a large number of new applications and services demand, operators have deployed large-scale network infrastructures to provide users with ubiquitous network service. Meanwhile, different radio access networks such as cellular networks, WLAN, and wireless mesh networks coexist in the free space around us forming a complex heterogeneous network environment. As a result, huge traffic and heterogeneous characteristics make the management of wireless networks difficult. In addition, with the increase of mobile service vendors and available bandwidth of wireless networks, users' requirements for performance of wireless networks and mobile services have become more and more trenchant. Mismatch between trenchant user requirements of perceived network quality for networks and difficult network management leads to great challenges in wireless networks.

However, wireless networks are traditionally designed with network-centric approaches. Different radio access technologies (RATs) have different resource formats and quality of service (QoS) strategies, and wireless networks are managed and optimized with the goal of improving network performance. Unfortunately, best network performance does not mean best user perceived quality of network service. Coexisting HetNets and QoS driven network management make wireless networks difficult to meet trenchant user requirements for network services. To address this problem brought by network-centric approaches, user-centric 
framework emerges as a disruptive new communication paradigm. User-centricity means the network is designed and built with the network users in the center to satisfy their requirements and preferences, which refactors the traditional wireless network models due to its user-centricity.

In terms of the user-centricity of proposed framework, two fundamental characteristics are indispensable to assuring user perceived quality in HetNets. Firstly, user-centric framework is supposed to provide users with ubiquitous and undifferentiated network connectivity, and all kinds of wireless networks should be scheduled uniformly to serve users. Users do not need to distinguish different RATs, as unified radio resources are provided for them. When users hand off between HetNets, seamless mobility could be achieved. Secondly, assurance of users' QoE for network services serves as the primary principle of user-centric resource management. Heterogeneous resources should be allocated dynamically according to user perceived quality in real time. Therefore, the fundamental principles related to user-centric wireless networks include ubiquitous and undifferentiated network connectivity and QoE assurance by effective resource and traffic management. However, how to design an integrated user-centric framework for heterogeneous wireless networks is still an open question.

Fortunately, the emergence of SDN proposes a possible solution for user-centric design for wireless networks. Meanwhile, network resource virtualization has emerged as a powerful technique for customized resource provisioning in wireless networks. Through virtualization based SDN approaches, user-centric design could be implemented. In this paper, we propose a user-centric framework for heterogeneous wireless networks based on SDN and virtualization. User agent corresponding to each user could be generated on soft access devices, which possess ability of connection information keeping and user situation awareness. Ubiquitous and undifferentiated network connectivity for users in heterogeneous wireless networks could be achieved by dynamic mitigation of user agent, and QoE assurance for users by flexible resources and traffic management could be achieved in the light of user situation awareness of user agent.

The remainder of this paper is organized as follows. We summarize the related work in Section 2. User-centric framework for heterogeneous wireless networks is described in Section 3. Ubiquitous and undifferentiated network connectivity and QoE assurance by flexible resources and traffic management are depicted in Sections 4 and 5, respectively. Performance of proposed user-centric framework and possible overhead is analyzed in Section 6, and the paper is concluded in Section 7.

\section{Related Work}

Generally, user-centricity is a key aspect of user-centric framework [2]. Researchers have introduced user-centric design to wireless networks in two aspects, such as usercentric radio resource management and user-centric service performance optimization. In the aspect of user-centric radio resource management, a user-centric adaptive clustering method [3] for coordinated multipoint transmission in dense cellular networks is described, and normalized outage capacity of each mobile station is maximized. A user-centric intercell interference coordination strategy [4] is proposed for downlink small cell networks. Each user selects the coordinating base stations (BSs) based on the relative distance between the home BS and the interfering BSs, and the dominant interference for each user is effectively identified and mitigated. A user-centric downlink cooperative transmission scheme [5] with orthogonal beamforming based limited feedback is proposed, and the percentage of users with satisfactory QoS demands is significantly increased. In the aspect of user-centric service performance optimization, user-centric QoE function [6] is modeled by a sigmoid function. Users' satisfaction on wireless services could be incorporated into the scheduler, and the average number of satisfied users is maximized. A QoE-driven usercentric solution for video on demand services [7] in urban vehicular network environments is introduced, and high QoE service level is provided to vehicle passengers. A user-centric mobile cloud computing service model [8] is presented, and the increasing demands from mobile users in terms of services diversity, user experience, security, and privacy could be met. Unfortunately, there is no work focusing on the user-centric design for heterogeneous wireless networks from resource management level to service performance optimization level. As heterogeneous wireless networks are built separately and have various radio resource formats, a user-centric framework will be a tough challenging issue.

On the other hand, to make the architecture of wireless networks more flexible, flattening, and programmable, the traditional wireless networks trend to combine with the concept of SDN. With the development of soft baseband and resource virtualization [9], this research field has attracted more and more attention in recent years. An SDN-enabled architecture for converged networks [10], which builds on the decoupling of data and control functionalities in the radio access network and control and forwarding functionalities in the core network, is proposed. Efficient resource management, QoS enforcement, and flexibility and scalability for future network evolution could be achieved. The authors present software defined access (SDA) [11], which introduces a novel logical control path across radio interfaces and up to mobile devices. Unlike SDN and SDWN (Software Defined Wireless Network), SDA can be deployed without changing network elements of radio access technologies. To deal with the increasing complexity in heterogeneous mobile networks (HMNs), authors believe that SDN based control is a promising approach to solve control problems in HMNs. An SDN based control framework named SoftMobile [12] to coordinate complex radio access in HMNs is proposed. In [13], all-SDN network architecture with hierarchical network control capabilities is advocated to allow for different grades of performance and complexity in offering core network services and provide service differentiation for $5 \mathrm{G}$ systems. In brief, SDN can be used to solve many challenging problems in wireless networks. Advanced wireless resources management in wireless networks, such as load balancing and coordination of inter-RAT basestation/APs, could be achieved through 


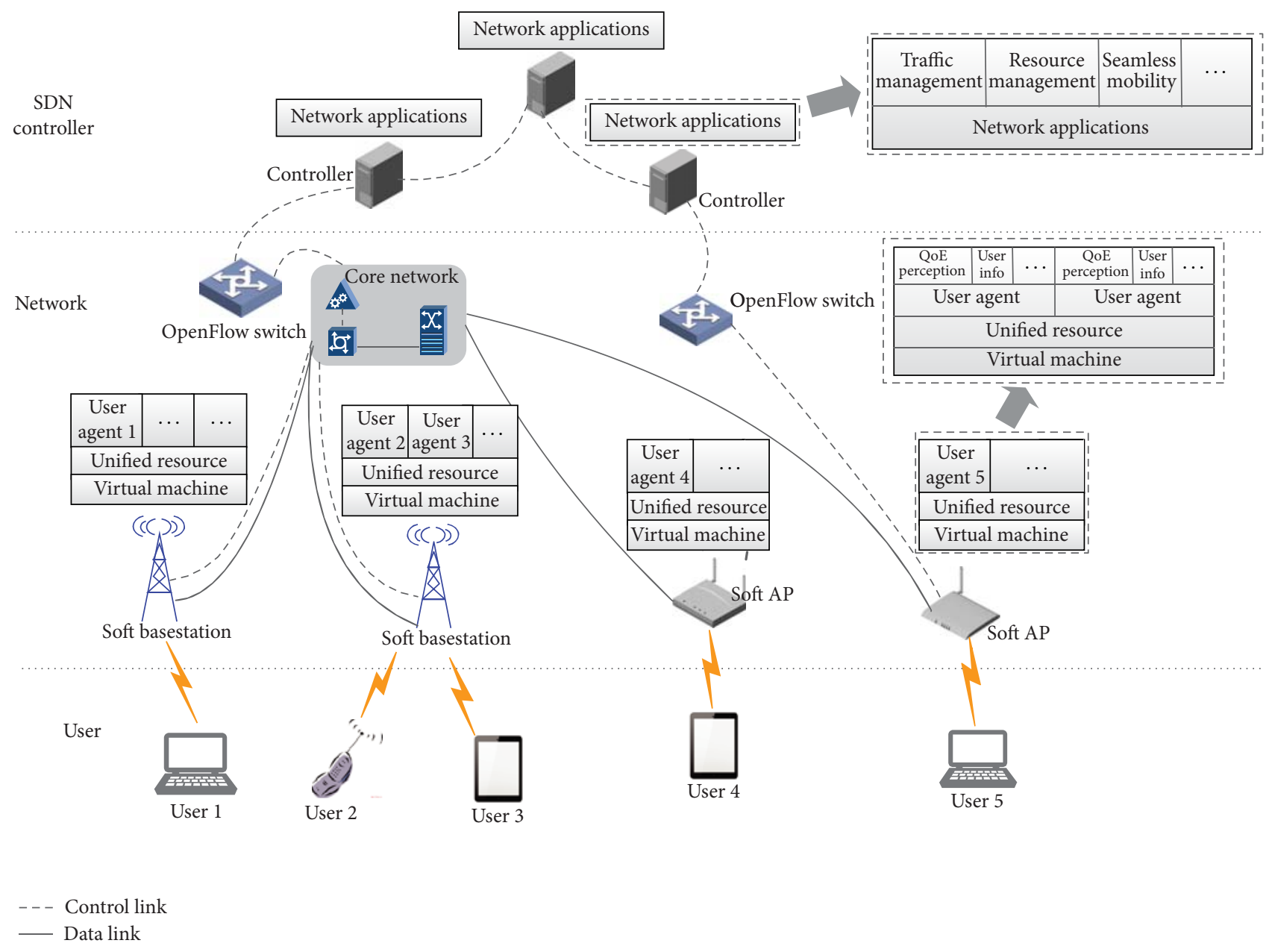

FIGURE 1: SDN based user-centric framework for wireless networks.

overall view of the controller. To operate wireless networks in a more efficient way, network resource virtualization may be a perfect method to work with SDN. Therefore, network frameworks coupling SDN with virtualization could be a pragmatic approach for user-centric design. Current wireless network virtualization researches, such as $[14,15]$, mainly focus on the sharing mechanism, including access infrastructure sharing and network sharing. In [16], the authors mention the virtualization of physical radio resources by adopting \{base station index, time, and frequency\} to abstract the radio resources. Authors illustrate the framework for combining SDN and wireless network virtualization and discuss the challenges for future study in [17]. However, no research work has attempted to design a user-centric framework by SDN, and several important issues should be focused on to reach this target. In this paper, we present a set of programming abstractions modeling the fundamental aspects of a wireless network, namely, user agent abstraction, user situation awareness, and resource and traffic management. The proposed abstractions hide away the implementation details of the underlying wireless technology and provide users with ubiquitous and undifferentiated network connectivity.

\section{User-Centric Framework for Heterogeneous Wireless Networks}

In order to accommodate the explosive mobile data traffic growth, current network operator may operate several wireless networks with different technologies simultaneously. Those heterogeneous networks are completely designed by network-centric approaches, built separately and mainly implemented by hardware which makes heterogeneous network management functions such as ubiquitous and undifferentiated network connectivity and QoE assurance for mobile users unprocurable. However, implementation of user-centric wireless networks needs a highly concentrated and flexible control plane to manage the heterogeneous underlying resources according to user's QoE. As depicted in Section 1, network resource virtualization technology and SDN will be two keys to resolve these issues. An SDN based user-centric framework for heterogeneous networks is depicted in Figure 1, and the network components of this proposed framework are composed of soft basestation/AP, virtual machine, user agent, OpenFlow switch, controller, and network applications. 
Soft basestation/AP is software defined radio (SDR) based basestation/AP whose baseband part is implemented by software. It provides users programming interfaces by which user agents can obtain user state information, link state information, types of users' services, and so on. To implement the soft basestation/AP, we adopt soft baseband and soft MAC [18] technologies, so that eNodeB in LTE, basestation in WiMAX, and AP in WLAN could be realized by general purpose processor (GPP) and software radio peripheral (either universal or exclusive).

Virtual machine (VM) realizes the function of the network resource virtualization. It has the advantage of network status awareness which includes physical layer perception and network layer measurement. The information perceived in physical layer refers to signal strength, interference, spectrum usage, and so on. And the network layer information includes connectivity, throughput, bandwidth, delay, jitter, and packet loss rate. Leveraged by the information, the VM could provide a mapping from heterogeneous wireless resources to unified virtualized resource elements with uniform format and informs controller of the usage situation of these unified virtualized resources. We use a plugin mode [19] to develop resource virtualization modules for each RAT.

User agent is an agent for users operating on the soft basestation/AP. It holds the user information for a certain user who is connected to the network and calculates the QoE of network service for this user. It uses the underlying virtual resources according to the schedule instructions of controller. When a user switches from one basestation/AP to another, the corresponding user agent also migrates from original basestation/AP to the corresponding one. When the traffic management function requires traffic of one user transmitted through two different basestation/APs, a new user agent will generate in the collaboration basestation/AP, which means that there will be two user agents operating on two corresponding basestation/APs for one specific user. The user agent is implemented by maintaining a configuration table in soft basestation/AP.

OpenFlow switch is a switch supporting OpenFlow protocol. It communicates with controller via OpenFlow protocol and executes the control instructions issued by the controller, and traffic management can be carried out by dynamic routing policy.

Controller is the core of the user-centric framework. It has a global view of the underlying network and can obtain the entire virtual resources of the whole network and all the available virtual resources information from the VMs, as well as user state, link state, traffic state, and QoE information from user agents. Traffic control messages are generated by controller and sent to OpenFlow switches to manage the user's traffic. Resource control messages are also generated by controller and sent to user agents so as to delete user agent, generate user agent, and manage the virtual radio resource. In addition, the controller provides open APIs which could be used by network administrators to develop network management applications and implement user-centric network functions.

Network applications include user-centric applications operating on the controller, such as traffic management,

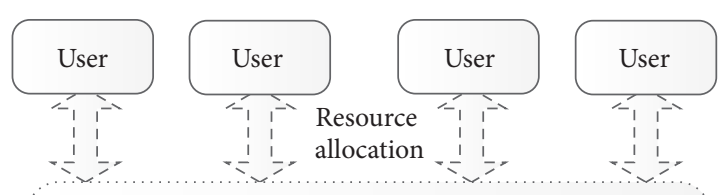

Unified virtual resources (bandwidth, time, and location)

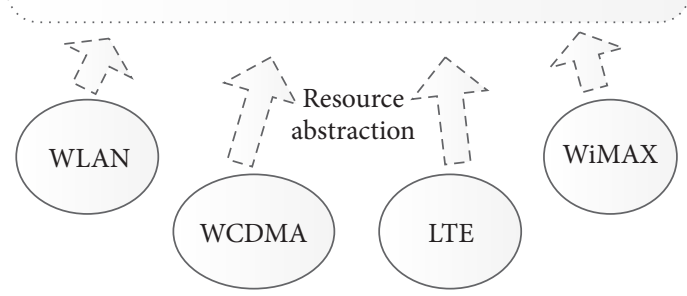

FIGURE 2: Resource abstraction and resource allocation in usercentric framework.

resource management, and seamless mobility. These applications are implemented through open APIs provided by the controller. Therefore, they are easy to develop and modify without any hardware changes.

Through SDN and network resources virtualization, the proposed framework changes the network management methodology from network-centric to user-centric, which thoroughly refactors the traditional wireless heterogeneous networks to a more flexible and coadjutant network. As a result, assurance of user perceived network qualities such as ubiquitous and undifferentiated network connectivity and QoE assurance of network services could be achieved.

\section{Ubiquitous and Undifferentiated Network Connectivity}

User-centric framework is supposed to provide users with ubiquitous network connectivity, and all kinds of RATs should be scheduled uniformly to serve users in the form of collaboration. In the proposed user-centric framework, heterogeneous wireless resources are abstracted to unified virtualized resources. Different kinds of wireless access methods have different kinds of physical radio resources, including power, spectrum, time, space, and code. By resource abstraction, a virtual resource pool that maintains the available resources comes into being in the heterogeneous wireless networks. As shown in Figure 2, we abstract the physical radio resource as a $3 \mathrm{D}$ resource grid: bandwidth, time, and location [19]. This is a location based resources abstraction, and it represents, at a specific time and location, how many bandwidth resources could be used by a corresponding user. With this metaresource model, the wireless resources are supposed to be expressed in a format directly corresponding to user's requirements.

To support the ubiquitous network connectivity for users in HetNets, seamless mobility among different RATs is another significant issue that should be addressed. Usercentric wireless networks is expected to give network users the ability to get undifferentiated connectivity to a certain point over the access network that best suits his or her current needs at any point in time. Seamless mobility is 
one fundamental function for our proposed user-centric framework. It is implemented by a network management application running on the controller and leveraged by the network resources virtualization technology. The introduction of user agent makes the connection status between user and soft basestation/AP controllable. User agent holds user information of multiple layers for a certain user which could help maintain the user's connection status. From user's perspective, an agent is a general basestation or AP that handles regular communication handshakes corresponding to this specific user. Just like virtual machines in data center can be migrated between different physical servers, user agents can migrate between different soft basestation/APs. If the user agent migrates as fast as the corresponding user's movement, a virtual persistent connection can be maintained for this user. That is, when one user switches from one soft basestation/AP to another, time delay caused by handover just includes time delay of break and reconnection in physical layer rather than time delay of multilayers such as physical layer, MAC layer, and network layer. Therefore, dynamic migration of user agent can be utilized to realize seamless mobility.

Specifically, resources virtualization and virtual machine migration technologies make unified radio resources and seamless mobility easy to implement. As previously described, heterogeneous wireless resources are abstracted into unified virtualized resource in a location based virtual resource format. Meanwhile, the controller, who has a global view of the virtual resources, can acquire user's location information, physical layer information such as signal strength, signal-noise ratio (SNR), and network layer information such as link throughput, delay, and packet loss rate from VM. Hence, the controller can perceive user's movement and predict the moving direction of mobile user. Then a variety of handover decisions algorithm which makes switching decisions based on different strategies [20] could be applied. If the handover conditions are met, the controller will migrate the user's agent from the source basestation/AP to the destination basestation/AP that the user is moving to, and seamless mobility of users could be achieved.

\section{QoE Assurance by Flexible Resources and Traffic Management}

The proposed user-centric framework aims at refactoring wireless mobile networks, and network management methodology is changed from network-centric to usercentric. Through migration of user agent, one virtual persistent and undifferentiated connection can be maintained for one specific user, which is the foundation of usercentric framework. Further, as user agent operating in soft basestation/AP is a virtual proxy of user and the user situation of mobile users can be aware, the controller can manage the traffic and virtualized resource aiming at best QoE for users. Hence, user-centric network functions such as flexible resource management and fine-grained traffic management for users could be achieved, and user-centric framework from resource management level to service performance optimization level could be implemented.

5.1. User Situation Awareness. In the proposed user-centric framework, user agent in soft basestation/AP calculates the QoE of network services for users and proactively sends this user's situation to the controller. By putting QoE as the optimization objective of network management, network design philosophy has been transferred to user-centric. Controller executes network operations including virtual resources management and traffic management according to a certain criterion to enhance user's QoE. This is a negative feedback procedure, and different strategies could be adopted by controller to realize QoE assurance of different granularity.

The goal of user situation awareness is to acquire the QoE information related to the specific user. Since different types of network services have different characteristics, in order to perceive user's QoE more accurately, mean objective score (MOS) is used as a uniform metric, while different wireless services are built by different QoE perception models. For example, for best effort (BE) services, we explore the role of human cognition and the psychophysics method in QoE assessment and establish a model in terms of the service information, complete time, and bandwidth to figure out QoE. For mobile video services, acceptability based quality assessment methods are adopted to perceive the QoE [21]. For different mobile video services, different feature vectors are chosen, and corresponding mapping approaches are adopted to calculate the MOS of video services.

In order to utilize the radio resource more effectively and enhance the user perceived service quality, user-centric function takes advantage of the broadcast nature of the wireless media by means of cooperative approaches such as flexible resource management and fine-grained traffic management.

5.2. Flexible Resource Management. Flexible resource management is the main user-centric function for heterogeneous radio resources, which manages heterogeneous radio resources of wireless networks by dynamic tuning the radio resource in soft basestation/AP, with QoE being the objective.

The virtualization and abstraction of heterogeneous wireless resources make the management of underlying physical resources as flexible as possible. These unified virtual resources are allocated to mobile users according to their requirements by optimization algorithms, such as auction algorithm, games theory, and water-filling algorithm. Resource management application is implemented in the controller as shown in Figure 3, which mainly consists of three components: status collection, decision making, and issuing instruction. Before making any decisions, controller will collect and update the underlying network status information, which includes the available virtual resources and user's QoE. Then, controller will execute decision making component which determines the virtual resource allocation scheme in each time slice. As soon as the allocation scheme is determined, controller will issue control instructions to user 


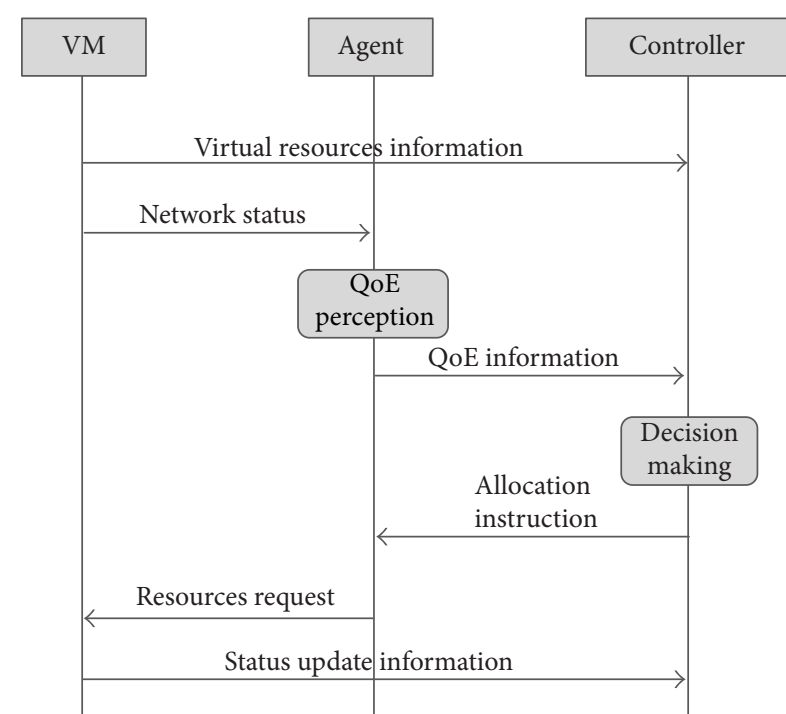

FIGURE 3: Flexible resources management in user-centric framework.

agent, who will take up underlying virtual resources held by VM according to the control instructions of controller.

5.3. Fine-Grained Traffic Management. Fine-grained traffic management is the user-centric function for network traffic, which manages the network traffic of HetNets by dynamically adjusting the traffic path and flexible basestation/AP selection with QoE as the objective. Traffic management is a comprehensive network application composed of flow table management, user agent generation/deletion, and data stream merging schemes. The controller can find the optimal routing path for user's traffic according to different strategies. OpenFlow switches in the network consist of one or more flow tables which perform packet lookups and forwarding. The controller communicates with the switch via the OpenFlow protocol to add, update, and delete flow tables reactively or proactively. Meanwhile, user agent receives the user data from OpenFlow switches and transfers it to user equipment through the underlying hardware infrastructure using corresponding packet format of different RATs. This mechanism makes the multicell cooperative transmission based traffic management possible. That is, when a user is in the overlapping coverage area of multiple basestation/APs, controller can issue instructions to generate multiple agents on these basestation/APs for this user, and data stream of services could be transmitted to user equipment from multiple paths. Data stream merging schemes are adopted by user equipment to combine streams transmitted by different paths. In user-centric wireless networking, network user's equipment are expected to support multiple RATs and treat the multiradio HetNets as a single network, which creates rich opportunities for intelligently combining and aggregating capacity across these RATs. That is, multiple RATs serve users in a collaborative manner to satisfy the requirements and preferences of network users.
As Figure 4 shows, eNodeB $A$ is heavily loaded while AP $A$ and AP $B$ serve few users. UE 1, who is served by eNodeB $A$ with a bad link state, is located at the overlapping area. In the user-centric design, UE 1 can support multiple RATs and establish a connection with AP A to offload partial traffic from eNodeB $A$. This procedure should be completely automatic and bring no interruption for network services.

\section{Performance and Overhead Analysis}

To prove the performance of the propose user-centric framework in heterogeneous wireless networks, a test bed is set up according to Figure 5. It is consisted of LTE (based on 3GPP release 10) and WLAN. Specifically, the LTE network consists of a core network and two soft basestations, which are implemented based on OpenAirInterface project [22]. The WLAN is implemented based on our existing SWAN experimental network [23]. Ten soft APs are deployed in this test bed, and each AP is equipped with wireless NICs working specifically in channel 6 (2.4 GHz band). The operating system of these APs is the OpenWrt "Backfire 10.03.1" release. Both basestations and APs are implemented by software on general purpose processor (GPP) platform and software radio peripheral. The network controller is a ThinkServer RD640 server equipped with a six-core Xeon E5-2620 CPU and 16 GB of RAM. iPerf is used for synthetic traffic generation.

Figure 6 shows the throughput over time of a user in the proposed framework and traditional heterogeneous network. For the traditional heterogeneous network, the throughput drops to zero for several seconds due to the user's mobility. However, in user-centric framework, users will perceive a stable connectivity in the handover process and the throughput curve remains uninterrupted with the user's mobility. As the user agent migrates with the corresponding user's movement, user can always see the consistent user agent connectivity regardless of the associated physical AP or basestation. The connectivity of users in user-centric based heterogeneous wireless networks will not break off. Therefore, the proposed framework can provide mobile users with continuous and consistent connectivity.

To demonstrate the performance of $\mathrm{QoE}$ assurance based on flexible resources and traffic management, user satisfaction, which denotes the ratio of the number of users who are satisfied with their QoE to the total number of users, is defined as

$$
\text { USER SATISFACTION }=\frac{\text { USER NUM }\left.\right|_{\text {MOS }>3.5}}{\text { TOTAL NUM }} .
$$

In our test bed, users with MOS value more than 3.5 are regarded as users satisfied with their QoE of services. Two most major services for users in wireless networks such as video streaming services and best effort services are considered in this test. Figure 7 depicted the user satisfaction of the proposed method, cross-layer QoE-aware based method [24], and proportional fairness based method. HetNets is composed of LTE and WLAN in this test. It is shown that the proposed method always maintains a high and stabilized user satisfaction with the increasing number of users, while the user satisfaction of QoE-aware based algorithm declines 


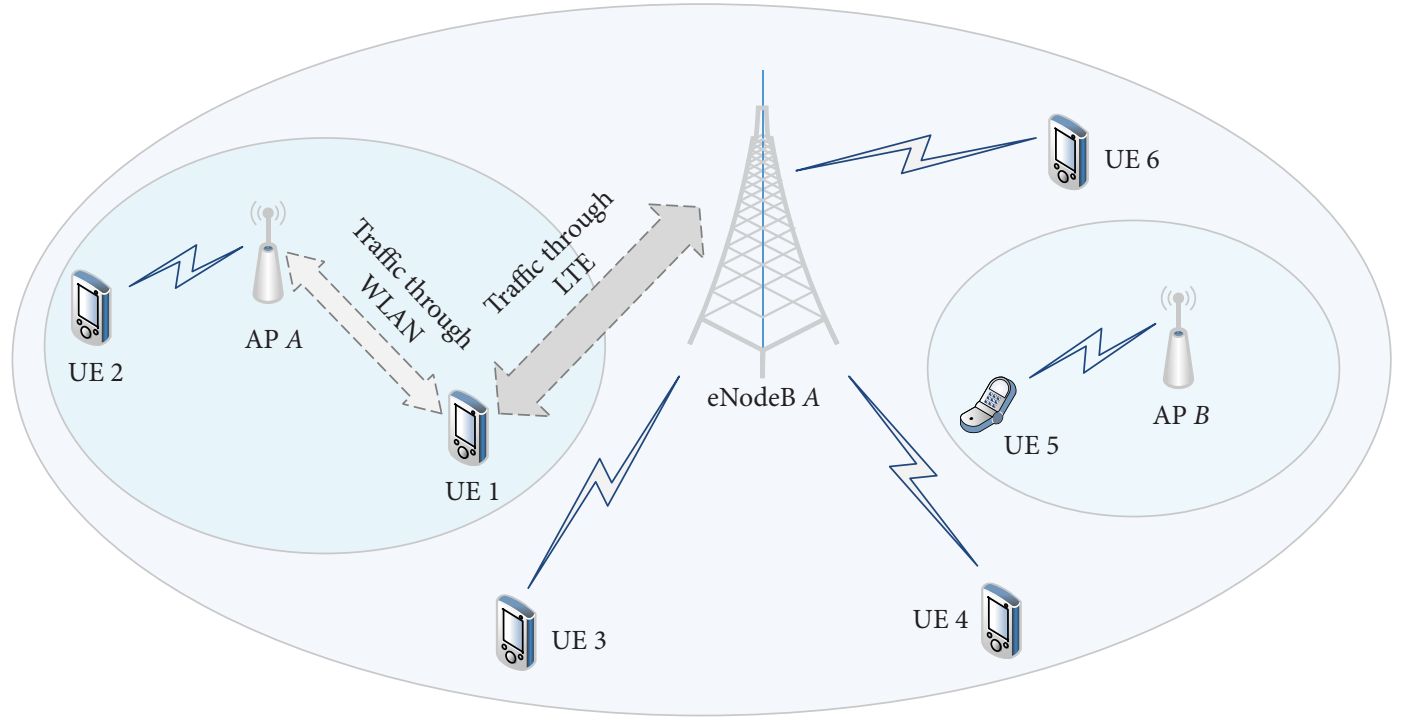

FIGURE 4: LTE cooperation with WLAN in user-centric framework.

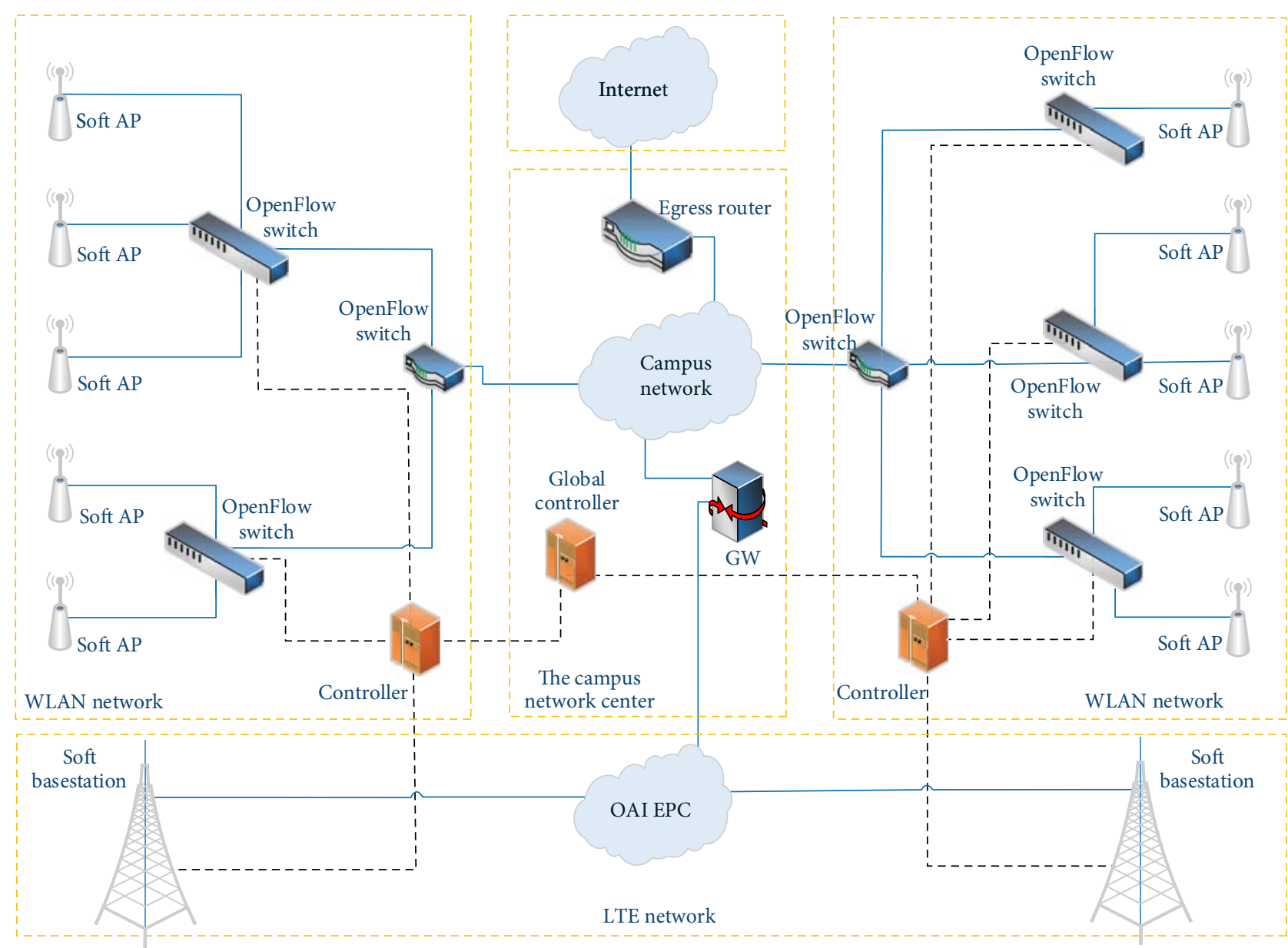

--- Control channel

_ Data channel

FigURE 5: The test bed for heterogeneous wireless networks. 


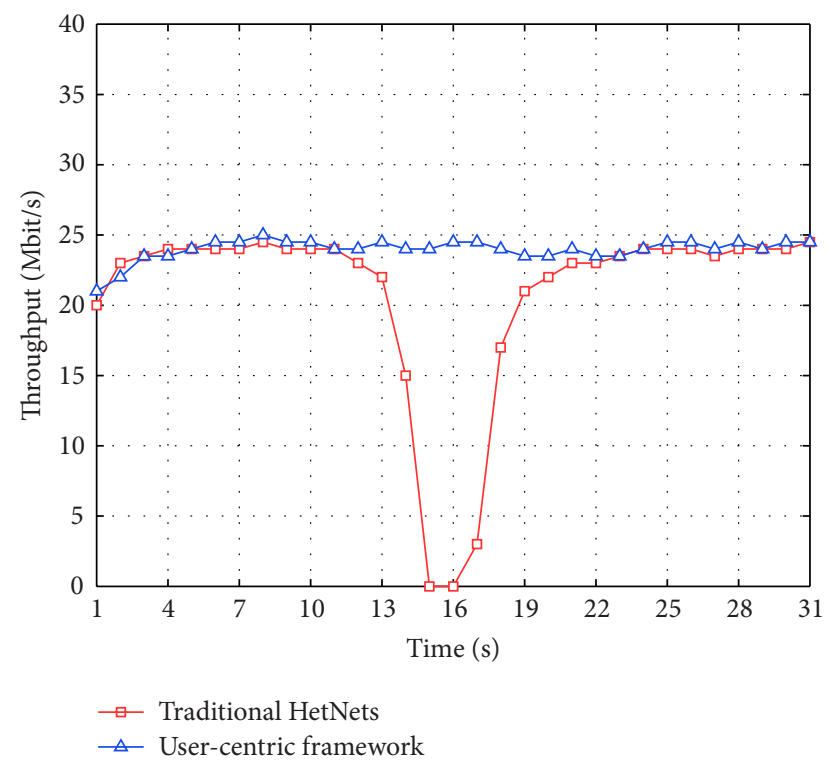

FIGURE 6: Seamless mobility for user-centric framework in heterogeneous wireless networks.

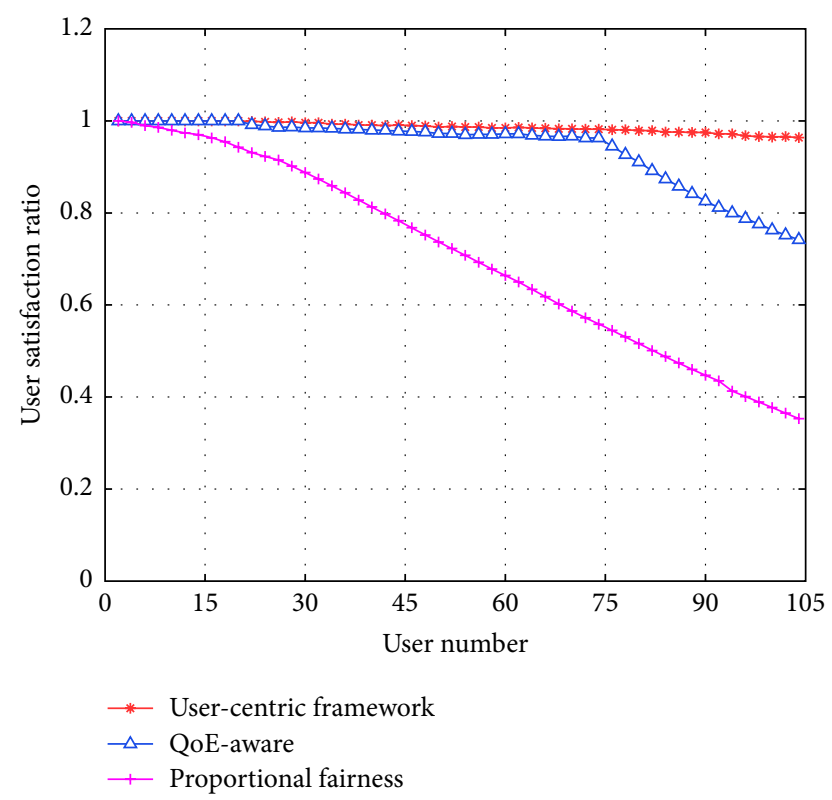

FIgURE 7: The user satisfaction of different resource allocation methods.

sharply when the user number exceeds a certain value when network capacity of LTE or WLAN is achieved. Besides, the curve of proportional fairness based algorithm gradually declines with the increasing number of users. Therefore, the proposed method can ensure users' QoE by flexible resources and traffic management.

Our proposed framework is built by the QoE metrics, namely, the intention to achieve more profit for users from the perspective of user-centric, while at the same time sharing the limited radio resources more effectively. Hence, algorithms and strategies proposed under this user-centric framework may be evaluated by QoE metrics in the future. Due to the user-centric framework design in wireless networks, QoE metrics could contribute a more dynamic behavior, while some costs that might be brought by SDN and resource virtualization, such as signaling overhead and control delay, are also investigated in this framework.

Signaling and Processing Overhead. With a global network awareness and management, the controller should perform dynamic network status awareness and virtualized resource management, which will generate the amount of signaling and processing overhead in controller. To solve this defect, hierarchical control plane could be adopted in this framework, by which domain controller and global controller appear to specialize in handling various tasks. Besides, efficient signaling compression and aggregation schemes could be used in this user-centric framework.

Control Delay. SDN makes a separation between the control plane and data plane in heterogeneous wireless networks. The virtualization of radio resources is accomplished on the data plane while the virtualized resources allocation is implemented on the control plane. As the wireless networks are highly time sensitive, the control signaling interaction between control plane and data plane may cause delays. Therefore, we argue that the data plane could be realized by a centralized base band processing pool as presented in CRAN, which will considerably shorten the control delay between control plane and data plane.

\section{Conclusion}

Network-centric framework in the dense HetNets scenario leads to a contradiction between trenchant user requirements and difficult management of the inflexible wireless networks. It is not able to provide network users with ubiquitous and undifferentiated network connectivity and QoE assurance. To address this issue, user-centric framework could be an effective solution. In this paper, we analyze the challenges and requirements for user-centric based heterogeneous wireless networks. Then, we propose a user-centric framework based on network resource virtualization technology and SDN for heterogeneous wireless networks. Ubiquitous and undifferentiated network connectivity and QoE assurance by flexible resources and traffic management are presented to implement the two fundamental characteristics of usercentricity. In addition, possible overheads of user-centric framework such as signaling overhead and control delay are also analyzed. As part of our ongoing work for the architecture of future wireless networks, we believe that this study can shed light on how we use SDN and virtualization technology to refactor future wireless network architecture and promote the research for $5 \mathrm{G}$ and beyond networks.

\section{Competing Interests}

The authors declare that they have no competing interests. 


\section{Acknowledgments}

This work is supported by Beijing Municipal Science and Technology Commission Research Fund Project no. D151100000115002 and the WLAN achievement transformation based on SDN Project of Beijing Municipal Commission of Education (Grant no. 201501001).

\section{References}

[1] D. Gilstrap, Ericsson Mobility Report, Ericsson, 2013.

[2] B. A. A. Nunes, M. A. S. Santos, B. T. De Oliveira, C. B. Margi, K. Obraczka, and T. Turletti, "Software-defined-networkingenabled capacity sharing in user-centric networks," IEEE Communications Magazine, vol. 52, no. 9, pp. 28-36, 2014.

[3] V. Garcia, Y. Zhou, and J. Shi, "Coordinated multipoint transmission in dense cellular networks with user-centric adaptive clustering," IEEE Transactions on Wireless Communications, vol. 13, no. 8, pp. 4297-4308, 2014.

[4] C. Li, J. Zhang, M. Haenggi, and K. B. Letaief, "User-centric intercell interference nulling for downlink small cell networks," IEEE Transactions on Communications, vol. 63, no. 4, pp. 14191431, 2015.

[5] D. Su and C. Yang, "User-centric downlink cooperative transmission with orthogonal beamforming based limited feedback," IEEE Transactions on Communications, vol. 63, no. 8, pp. 29963007, 2015.

[6] G. Lee, H. Kim, Y. Cho, and S.-H. Lee, "QoE-aware scheduling for sigmoid optimization in wireless networks," IEEE Communications Letters, vol. 18, no. 11, pp. 1995-1998, 2014.

[7] C. Xu, F. Zhao, J. Guan, H. Zhang, and G.-M. Muntean, "QoEdriven user-centric VoD services in urban multihomed P2Pbased vehicular networks," IEEE Transactions on Vehicular Technology, vol. 62, no. 5, pp. 2273-2289, 2013.

[8] D. Huang, T. Xing, and H. Wu, "Mobile cloud computing service models: a user-centric approach," IEEE Network, vol. 27, no. 5, pp. 6-11, 2013.

[9] Q. Yang, X. Li, H. Yao et al., "Bigstation: enabling scalable realtime signal processing in large mu-mimo systems," Proceedings of the ACM SIGCOMM Computer Communication Review (SIGCOMM '13), vol. 43, no. 4, pp. 399-410, 2013.

[10] W. Tan, J. Zhang, C. Peng, B. Xia, and Y. Kou, "SDN-enabled converged networks," IEEE Wireless Communications, vol. 21, no. 6, pp. 79-85, 2014.

[11] V. Sagar, R. Chandramouli, and K. P. Subbalakshmi, "Software defined access for HetNets," IEEE Communications Magazine, vol. 54, no. 1, pp. 84-89, 2016.

[12] T. Chen, H. Zhang, X. Chen, and O. Tirkkonen, "SoftMobile: control evolution for future heterogeneous mobile networks," IEEE Wireless Communications, vol. 21, no. 6, pp. 70-78, 2014.

[13] V. Yazici, U. C. Kozat, and M. O. Sunay, "A new control plane for $5 \mathrm{G}$ network architecture with a case study on unified handoff, mobility, and routing management," IEEE Communications Magazine, vol. 52, no. 11, pp. 76-85, 2014.

[14] R. Kokku, R. Mahindra, H. Zhang, and S. Rangarajan, "NVS: a substrate for virtualizing wireless resources in cellular networks," IEEE/ACM Transactions on Networking, vol. 20, no. 5, pp. 1333-1346, 2012.

[15] C. J. Bernardos, A. De La Oliva, P. Serrano et al., "An architecture for software defined wireless networking," IEEE Wireless Communications, vol. 21, no. 3, pp. 52-61, 2014.
[16] A. Gudipati, D. Perry, L. E. Li, and S. Katti, "Softran: software defined radio access network," in Proceedings of the 2nd ACM SIGCOMM Workshop on Hot Topics in Software Defined Networking (HotSDN '13), pp. 25-30, August 2013.

[17] B. Cao, F. He, Y. Li, C. Wang, and W. Lang, "Software defined virtual wireless network: framework and challenges," IEEE Network, vol. 29, no. 4, pp. 6-12, 2015.

[18] MAC80211, https://wireless.wiki.kernel.org/en/developers /Documentation/mac80211.

[19] L. Wang, Z. Lu, X. Wen, and W. Guan, "Converged management in heterogeneous wireless networks based on resource virtualization," Mobile Networks and Applications, vol. 20, no. 1, pp. 5361, 2015.

[20] A. Ahmed, L. M. Boulahia, and D. Gaït, "Enabling vertical handover decisions in heterogeneous wireless networks: a stateof-the-art and a classification," IEEE Communications Surveys and Tutorials, vol. 16, no. 2, pp. 776-811, 2014.

[21] W. Song and D. W. Tjondronegoro, "Acceptability-based QoE models for mobile video," IEEE Transactions on Multimedia, vol. 16, no. 3, pp. 738-750, 2014.

[22] OpenAirInterface Project, http://www.openairinterface.org/.

[23] T. Lei, Z. Lu, X. Wen, X. Zhao, and L. Wang, "SWAN: an SDN based campus WLAN framework," in Proceedings of the 4th International Conference on Wireless Communications, Vehicular Technology, Information Theory and Aerospace and Electronic Systems (VITAE '14), pp. 1-5, May 2014.

[24] M. Rugelj, U. Sedlar, M. Volk, J. Sterle, M. Hajdinjak, and A. Kos, "Novel cross-layer QoE-aware radio resource allocation algorithms in multiuser OFDMA systems," IEEE Transactions on Communications, vol. 62, no. 9, pp. 3196-3208, 2014. 

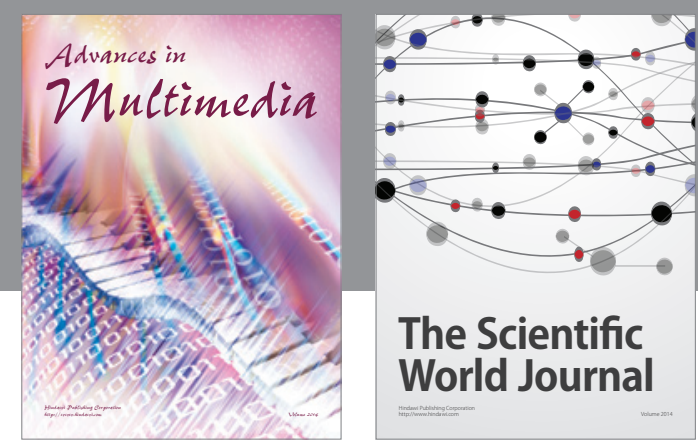

The Scientific World Journal
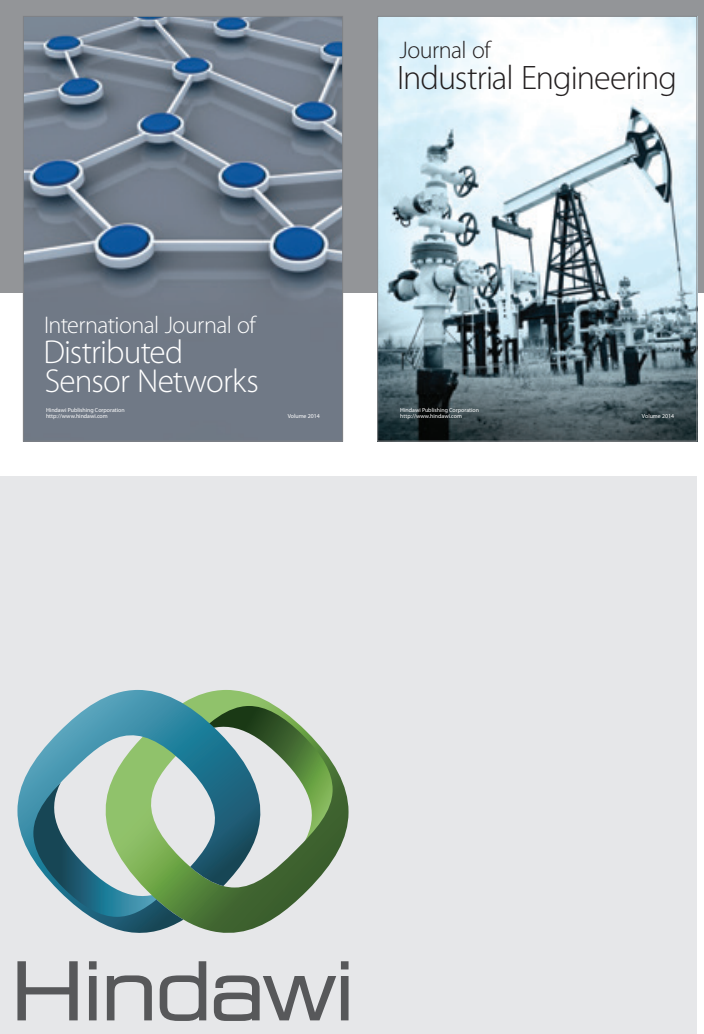

Submit your manuscripts at

http://www.hindawi.com

\section{Computer Networks} and Communications
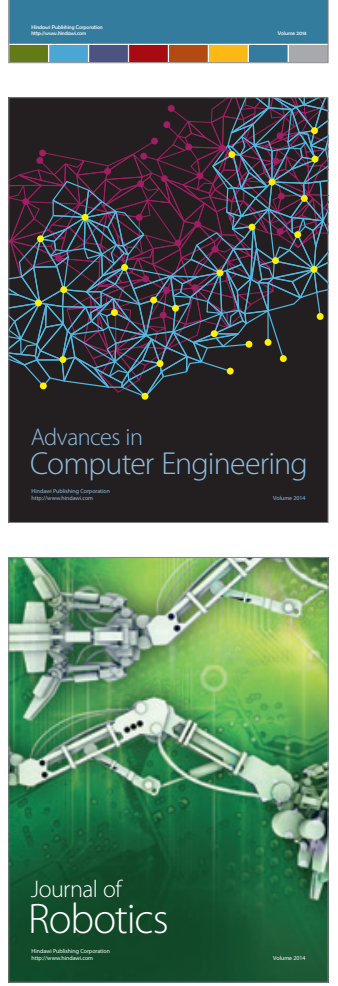
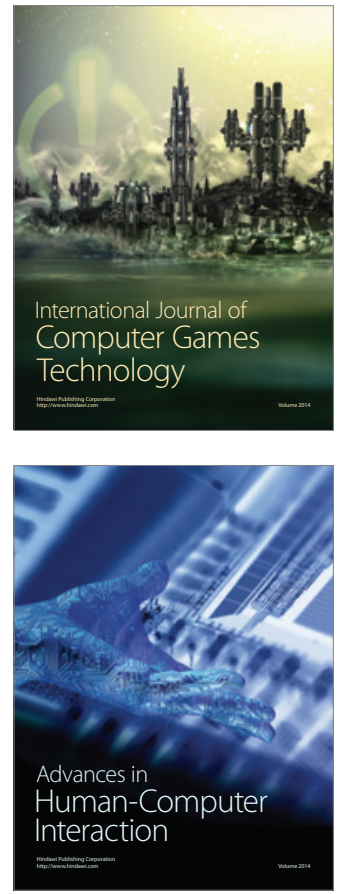
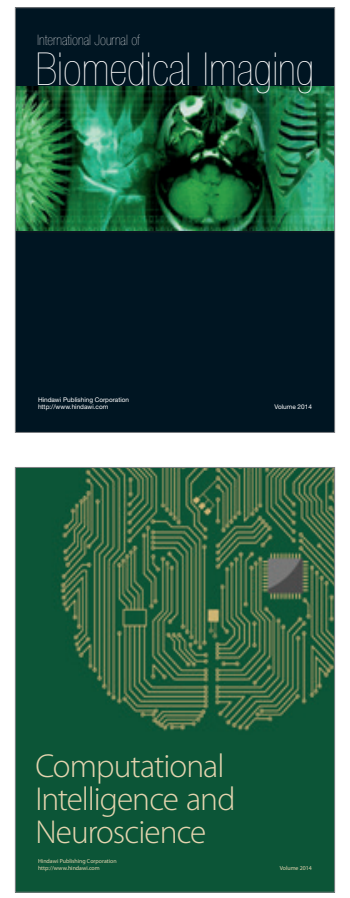
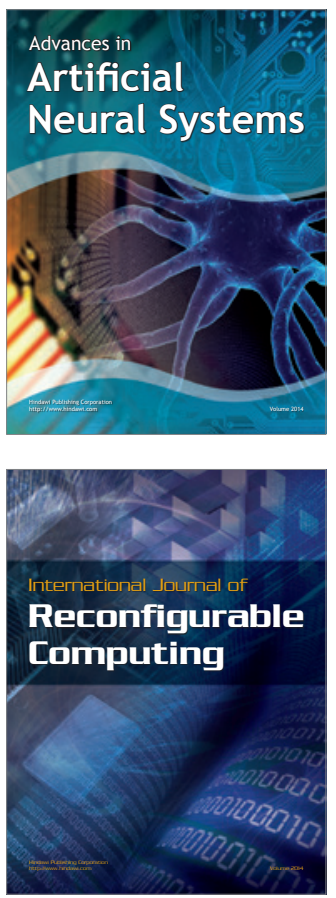
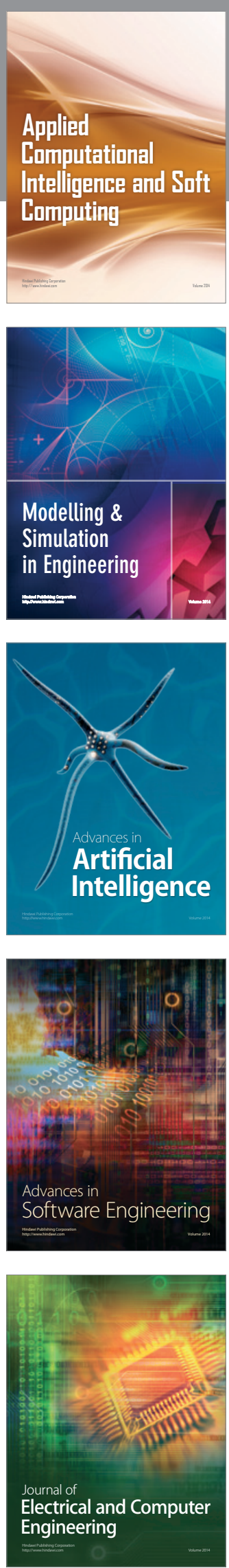